\title{
Investigation of folate-conjugated fluorescent silica nanoparticles for targeting delivery to folate receptor-positive tumors and their internalization mechanism
}

\author{
This article was published in the following Dove Press journal: \\ International Journal of Nanomedicine \\ 16 September 2011 \\ Number of times this article has been viewed
}

\section{Hong Yangl,* \\ Changchun Lou',** \\ Mingming $\mathrm{Xu}^{\prime}$ \\ Chunhui Wu' \\ Hirokazu Miyoshi \\ Yiyao Liu',3}

'Department of Biophysics, School of Life Science and Technology, University of Electronic Science and Technology of China, Chengdu, Sichuan, People's Republic of China; ${ }^{2}$ Radioisotope Research Center, University of Tokushima, Tokushima, Japan; ${ }^{3}$ Key Laboratory of Biorheological Science and Technology, Ministry of Education, Bioengineering College, Chongqing University, Chongqing, People's Republic of China

*These authors contributed equally to this work

\begin{abstract}
Multifunctionalized nanoparticles (NPs) are emerging as ideal tools for gene/drug delivery, bioimaging, labeling, or intracellular tracking in biomedical applications, and have attracted considerable attention owing to their unique advantages. In this study, fluorescent silica NPs were synthesized by a modified Stöber method using conjugates of 3-mercaptopropyltrimethoxysilane (MPS) and maleimide-fluorescein isothiocyanate (maleimide-FITC). Mean diameters of the NPs were controlled between 212-2111 nm by regulating MPS concentration in the reaction mixture. Maleimide-FITC molecules were doped into NPs or conjugated to the surface of NPs through the chemical reaction of maleimide and thiol groups. The data showed that the former NPs are better than the latter by comparing their fluorescence intensity. Furthermore, folate molecules were linked to the FITC-doped silica NPs by using polyethylene glycol (PEG) (NH2-PEG-maleimide) as a spacer, thus forming folate receptor targeting fluorescent NPs, referred to as NPs(FITC)-PEG-Folate. The quantitative analysis of cellular internalization into different cancer cells showed that the delivery efficiency of $\mathrm{KB}$ cells (folate receptor-positive cells) is more than six-fold higher than that of A549 cells (folate receptor-negative cells). The delivery efficiency of $\mathrm{KB}$ cells decreased significantly after free folate addition to the cell culture medium because the folate receptors were occupied by the free folate. The NPs endocytosis mechanism was also investigated. It was shown that clathrin, an inhibitor of cell phagocytosis, markedly decreased the NPs uptake into KB cells, suggesting that it plays an important role in NPs cellular internalization. These results demonstrated that the novel particles of NPs(FITC)-PEG-Folate are promising for fluorescent imaging or targeting delivery to folate receptor-positive tumors.
\end{abstract}

Keywords: fluorescent nanoparticles, silica, folate, targeted delivery, cellular internalization

\section{Introduction}

The application of nanotechnology to biomedical research is expected to have a major impact leading to the development of new types of diagnostic and therapeutic tools. ${ }^{1-3}$ Recently, one focus in nanobiotechnology has been the development and use of multifunctional nanoparticles (NPs), which can be used to map cellular components and monitor and track them in real time, and as drug delivery vehicles or therapeutic agents. ${ }^{4}$ Silica NPs are favored since silicone dioxide is a nontoxic compound present in many systems, which can be tailored with a variety of surface modifiers, allowing properties such as zeta potential and surface reactivity to be adjusted. Thereby, silica NPs are being formulated for potential drug delivery, imaging, and diagnostic applications.,
Correspondence: Yiyao Liu Department of Biophysics, School of Life Science and Technology, University of Electronic Science and Technology of China, Chengdu 610054, Sichuan, People's Republic of China

Tel +8628 83203353

Fax +862883208238

Email liuyiyao@hotmail.com 
As mentioned above, silica has been utilized extensively as a nanomaterial for biological applications because it provides both a surface for bioconjugation and a host matrix for fluorescent molecules that can improve dye photostability and biocompatibility. However, it is challenging to fabricate fluorescent NPs or nanoprobes with high photostability, high payloads of dyes, and a desirable outer surface for further modification with functional or target moieties. To this end, at least two principles or strategies should be considered. One is that as many fluorescent molecules as possible should be doped into the silica NPs to minimize leakage from the NPs during applications. The other is the development of suitable surface residues for further modification. Recently, Nakamura and coworkers have synthesized several types of silica nanoparticles using various silica sources, and a fluorescent dye was imposed on the silica network via a succinimidyl ester reaction. These multifluorescent silica NPs were effectively applied in flow cytometry and fluorescent microscopy analyses..$^{7-9}$

Avoiding nonspecific delivery is another important concern in the design of NPs. In many papers it has been reported that insufficient uptake at tumor sites will decrease the therapeutic benefit of the administered drug dose and the affectivity of imaging, and that nonspecific association with healthy tissues can lead to toxic side effects. ${ }^{10-13}$ One strategy to enhance the cellular uptake (or internalization) of NPs is the utilization of unique molecular markers that are specifically overexpressed within cancerous tissues, particularly for receptor-mediated endocytosis using specific ligands to recognize their specific receptors on the cell membrane. It is well known that many malignant tissues consistently express high levels of folate receptors, which have been presented as an attractive target for tumor-selective drug delivery and imaging through the specific combination with relevant ligands of folate molecules. ${ }^{12,14-17}$

Here, we report a new class of biomultifunctional NPs, $\mathrm{NPs}$ (fluorescein isothiocyanate [FITC])-polyethylene glycol (PEG)-Folate, for targeting delivery and fluorescent imaging with a mean diameter of $212 \mathrm{~nm}$ that use 3-mercaptopropyltrimethoxysilane (MPS) as the unique silica source. FITC molecules were doped into NPs, and thiol group residues were presented on the nanoparticle surfaces. Folate molecules were further conjugated to the NPs surface by using modified PEG (NH2-PEG-maleimide) as a spacer. These novel silica NPs (NPs[FITC]-PEG-Folate) have two functions for targeted cancer cells, ie, ligand biorecognition targeting (folate) and fluorescent tracking (FITC). These double-modality silica NPs are expected to provide an excellent platform for drug delivery, bioimaging, and tracking for folate receptor positive cancer cells in the future.

\section{Materials and methods Materials}

3-MPS, dicyclohexylcarbodiimide (DCC), N-hydroxysuccinimide (NHS), sodium azide, and nystatin were obtained from Sigma-Aldrich Co (St Louis, MO). Maleimide-FITC (maleimide-FITC) was purchased from Vector Laboratories (Burlingame, $\mathrm{CA}$ ). $\mathrm{NH}_{2}$-PEG-maleimide was supplied by Avanti Polar Lipids, Inc (Alabaster, AL). Folate was purchased from Alexis Biochemicals (San Diego, CA). Dimethyl sulfoxide (DMSO) was obtained from Amresco, Inc (Solon, OH). Chlorpromazine and cytochalasin D were from Enzo Biochem (New York, NY). The cell culture medium and fetal bovine serum (FBS) were from Invitrogen Corporation (Carlsbad, CA). All chemicals and reagents were of analytical grade.

\section{Preparation of silica NPs and fluorescent silica NPs}

Silica NPs were prepared as described in a previous study with a minor modification. ${ }^{7}$ Briefly, MPS and ammonia $(28 \%)$ were dissolved in deionized water $\left(\mathrm{ddH}_{2} \mathrm{O}\right)$ and magnetically stirred (500 rpm) for 3 days at room temperature under different conditions as shown in Table 1. The synthesized silica NPs were washed five times by centrifugation for 5 minutes at $1000 \times \mathrm{g}$. The washed silica NPs were resuspended in $\mathrm{ddH}_{2} \mathrm{O}$.

The silica NPs suspension $(500 \mu \mathrm{L})$ and $8 \mu \mathrm{L}$ of maleimide-FITC were mixed and magnetically stirred (200 rpm) in darkness for 2 hours. The FITC molecules were

Table I Formation of silica nanoparticles under conditions (I), (2), and (3) which involved stirring for 3 days at room temperature $\left(25^{\circ} \mathrm{C}\right)$

\begin{tabular}{lllllll}
\hline & MPS $(\mu \mathrm{L})$ & Ammonia $(\mu \mathrm{L})$ & $\mathbf{d d H}_{2} \mathbf{O}(\mu \mathrm{L})$ & Total volume $(\mu \mathrm{L})$ & MPS concentration $(\mathbf{m M})$ & Diameter $(\mathrm{nm})$ \\
\hline Condition (I) & 768 & 360 & 3872 & 5000 & 400 & $2111 \pm 159$ \\
Condition (2) & 192 & 360 & 4448 & 5000 & 100 & $508 \pm 50$ \\
Condition (3) & 24 & 360 & 4616 & 5000 & 12.5 & $212 \pm 34$ \\
\hline
\end{tabular}

Abbreviations: MPS, 3-mercaptopropyltrimethoxysilane; $\mathrm{ddH}_{2} \mathrm{O}$, deionized water. 
conjugated onto the surface of the NPs through the chemical reaction of the maleimide and thiol groups. After incubation, the reaction mixture was subjected to centrifugation at $1000 \times \mathrm{g}$ for 5 minutes twice with DMSO and three times with $\mathrm{ddH}_{2} \mathrm{O}$. The obtained FITC-surface-conjugating silica NPs (referred to as NPs-FITC) were resuspended in $\mathrm{ddH}_{2} \mathrm{O}$.

Silica NPs with internally doped FITC (referred to as NPs[FITC]) were synthesized as shown in Figure 1. First, a mixture of $24 \mu \mathrm{L}$ of MPS, $32 \mu \mathrm{L}$ of maleimide-FITC (20 mg/mL), and $100 \mu \mathrm{L}$ of DMSO was gently stirred at $100 \mathrm{rpm}$ for 24 hours. MPS reacted with maleimide-FITC to form MPS-FITC conjugates through the chemical reaction of maleimide and thiol groups (Figure 1A). Then $360 \mu \mathrm{L}$ of ammonia (28\%) and $4585 \mu \mathrm{L}$ of $\mathrm{ddH}_{2} \mathrm{O}$ were added, and the mixture was incubated with gentle mixing for 3 days ( $500 \mathrm{rpm})$ in darkness at room temperature. After incubation, the purification procedure used for preparation of NPs-FITC was performed. The NPs[FITC] were also resuspended in $\mathrm{ddH}_{2} \mathrm{O}$.

\section{Surface modification of fluorescent silica NPs}

Folate $(0.8 \mathrm{mg})$ dissolved in $\mathrm{ddH}_{2} \mathrm{O}$ was activated by adding DCC $(0.4 \mathrm{mg})$ and NHS $(0.4 \mathrm{mg})$, then gently stirred $(100 \mathrm{rpm})$ at room temperature for 30 minutes. The $\mathrm{NH}_{2}-$ PEG-maleimide derivative $(0.5 \mathrm{mg})$ was dissolved in DMSO and added to the folate solution. The reaction was carried out in the presence of $10 \mu \mathrm{L}$ pyridine at room temperature for 3 hours. The resulting conjugates were dialyzed against $\mathrm{ddH}_{2} \mathrm{O}$ and filtered through a $0.45 \mu \mathrm{m}$ filter unit (Millipore Research and Development, Bedford, MA) to remove remaining reagents. The maleimide-PEG-folate was mixed to NPs(FITC) in DMSO under an octafluoropropane atmosphere at room temperature for 6 hours, and conjugated to the surface of NPs(FITC) to form a novel type of NPs(FITC)PEG-Folate. The resulting product was dialyzed against $\mathrm{ddH}_{2} \mathrm{O}$ and lyophilized.

\section{Characterization of NPs}

The morphology and size of the NPs were evaluated by scanning electron microscopy (SEM). The NPs suspension was directly trickled onto a nitrocellulose membrane attachment 400-mesh copper grid and images were observed by SEM (JEM 100CX; JEOL Ltd, Tokyo, Japan) with an accelerating voltage of $30-80 \mathrm{kV}$. The average particle size of the NPs, based on the results for at least 20 particles, was obtained from SEM images by ImageJ software analysis (http:// rsbweb.nih.gov/ij/). The surface charge was measured by determining the zeta potential using a Zetasizer Nano ZS (Malvern Instruments Ltd, Malvern, UK). The suspensions of fluorescent silica NPs (NPs-FITC and NPs[FITC]) were further observed under an inverted fluorescence microscope (Eclipse TE2000U; Nikon Corporation, Tokyo, Japan) to verify the fluorescent dye conjugation and compare their fluorescent intensities.

A<smiles>CO[Si](CCCS)(OC)OC</smiles>

MPS
HO<smiles>Cc1ccc2c(-c3ccc(C(=O)NCCN4C(=O)C=CC4=O)cc3C(=O)O)c3ccc(=O)cc-3oc2c1</smiles>

Mal-FITC<smiles>CO[Si](CCCSC1CC(=O)N(CCNC(=O)c2ccc(-c3c4ccc(=O)cc-4oc4cc(O)ccc34)c(C)c2)C1=O)(OC)OC</smiles>

MPS-FITC

B

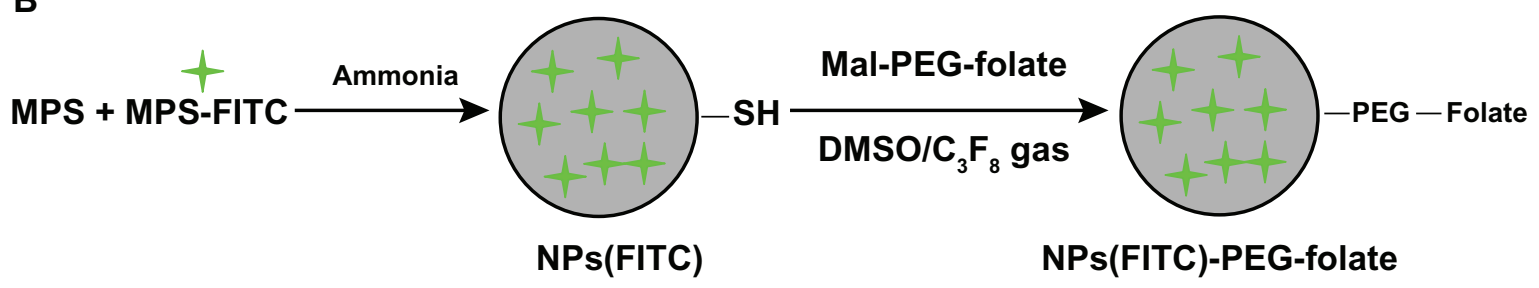

Figure I Schematic diagram of the preparation of nanoparticles-(fluorescein isothiocyanate)-polyethylene glycol-Folate (NPs[FITC]-PEG-Folate) particles. (A) The maleimide end of maleimide-FITC (Mal-FITC) reacts with thiol groups of 3-mercaptopropyltrimethoxysilane (MPS) to form stable thioester linkages. (B) Folate was activated with ethyl(dimethylaminopropyl) carbodiimide/ $\mathrm{N}$-hydroxysuccinimide and reacted with $\mathrm{NH}_{2}$-PEG-maleimide to form a reactive intermediate (maleimide-PEG-Folate), then the $\mathrm{NPs}(\mathrm{FITC})$ particles react with maleimide-PEG-Folate to produce NPs(FITC)-PEG-Folate particles.

Abbreviations: $\mathrm{C}_{3} \mathrm{~F}_{8}$, octafluoropropane; DMSO, dimethyl sulfoxide. 
The fluorescence spectroscopy of NPs(FITC) and NPs(FITC)-PEG-Folate suspensions was performed with an F-7000 Fluorescence Spectrophotometer (Hitachi HighTechnologies Corporation, Tokyo, Japan). The excitation and emission wavelengths were $280 \mathrm{~nm}$ and $440 \mathrm{~nm}$, respectively. The conjugation was further characterized by Fourier transform infrared (FT-IR) spectroscopy (Nicolet NEXUS 670 FTIR; Thermo Fisher Scientific, Waltham, MA) through a comparison of the absorbance spectra of NPs(FITC) and $\mathrm{NPs}$ (FITC)-PEG-Folate particles.

\section{Incubation of living cells with silica NPs}

Human epidermal carcinoma cells (KB cells, a folate receptor-positive cell line) and human lung carcinoma cells (A549 cells, a folate receptor-negative cell line) were grown in Dulbecco's Modified Eagle's Medium (DMEM) supplemented with $10 \%$ FBS and $1 \%$ penicillin-streptomycin, and cells were maintained in a humidified incubator at $37^{\circ} \mathrm{C}$ in $5 \%$ carbon dioxide and $95 \%$ air. $\mathrm{KB}$ or $\mathrm{A} 549$ cells were seeded onto glass coverslips in six-well plates at a density of $1 \times 10^{5}$ cells/well. Unless otherwise specified, the mean diameter of the NPs used in the following cellular experiments is $212 \mathrm{~nm}$ (Table 1, condition 3). Cell and NPs suspensions were incubated as follows: cells were cultured overnight (approximately 24 hours) to allow cell attachment and then washed with FBS-free DMEM. An NPs(FITC) or NPs(FITC)PEG-Folate suspensions was then added to each well and the resulting mixture was left to incubate at $37^{\circ} \mathrm{C}$ for 6 hours. The NPs concentration in the incubation culture was typically $480 \mu \mathrm{g} / \mathrm{mL}$. After incubation, the cells were washed several times with sterilized phosphate-buffered saline (PBS) before further examination under a fluorescence microscope.

\section{Confocal fluorescence microscopy}

The above-treated KB or A549 cells were fixed with $4 \%$ paraformaldehyde for 20 minutes at room temperature. Then the cells were washed three times with sterilized PBS to remove the remaining paraformaldehyde. The cells were further stained with a nuclei-specific dye, DAPI (Biyuntian Ltd Co, Beijing, China), at room temperature for 15 minutes, followed by washing three times with sterilized PBS. Finally, the stained cells were mounted for confocal microscope examination (Leica TCS SP5 II; Leica Microsystems, Wetzlar, Germany).

\section{Quantitative analysis of NPs uptake by flow cytometry}

To quantitatively determine the uptake efficiencies of the NPs, KB or A549 cells were seeded in 12-well plates at a density of $1 \times 10^{5}$ cells/well and allowed to grow for 24 hours before the treatment. The cells were washed three times with sterilized PBS, then $1 \mathrm{~mL}$ DMEM was added, and the mixture was incubated with $480 \mu \mathrm{g} / \mathrm{mL} \mathrm{NPs(FITC)} \mathrm{or}$ NPs(FITC)-PEG-Folate particles for 6 hours in an FBS-free medium. After incubation, the cells were washed repeatedly with sterilized PBS. The cells were then trypsinized and suspended in PBS for flow cytometry analysis (FACSCanto II; Becton Dickinson and Company, Franklin Lakes, NJ). For an experiment on free folate inhibition, free folate $(1 \mathrm{mM})$ was preincubated with KB cells for 1 hour before the incubation of NPs(FITC)-PEG-Folate.

\section{Endocytosis-inhibition experiments}

To investigate the mechanism of how NPs enter cells, a series of endocytosis-inhibition experiments was carried out. Cells were first pretreated with one of the endocytosis inhibitors $(0.1 \%$ sodium azide for the depletion of adenosine triphosphate (ATP); $10 \mu \mathrm{g} / \mathrm{mL}$ chlorpromazine for the inhibition of clathrin-mediated endocytosis; $15 \mu \mathrm{g} / \mathrm{mL}$ nystatin for the inhibition of caveolae-mediated endocytosis; or $1 \mu \mathrm{g} / \mathrm{mL}$ cytochalasin D for the inhibition of macropinocytosis) in FBS-free DMEM for 1 hour. Then the supernatant was discarded and the cells were washed three times with sterilized PBS. The cells in each well were resuspended in $1 \mathrm{~mL}$ DMEM and incubated with $480 \mu \mathrm{g}$ NPs(FITC) or NPs(FITC)-PEGFolate at $37^{\circ} \mathrm{C}$ for 6 hours. After incubation, the cells were rinsed three times with sterilized PBS, and flow cytometry analysis (FACSCanto II) was performed to examine the cellular internalization of the NPs.

\section{Presentation of data and statistical analysis}

Data obtained were represented as mean \pm standard deviation. Comparisons between groups were made by two-tailed Student's $t$-test using Windows Excel 2003 (Microsoft Corporation, Redmond, WA), and $P<0.05$ was used as the cutoff for defining statistically significant differences.

\section{Results and discussion Size and morphological characterization by SEM}

By using MPS as the sole silica source, silica NPs were synthesized successfully in accordance with the method reported by Nakamura and coworkers with a minor modification. ${ }^{7} \mathrm{NP}$ diameters were controlled between $212 \mathrm{~nm}$ and $2111 \mathrm{~nm}$ by controlling MPS concentration in the reaction mixtures. The morphology of the silica NPs was observed by SEM (Figure 2). It is clear that the silica NPs are uniform and 

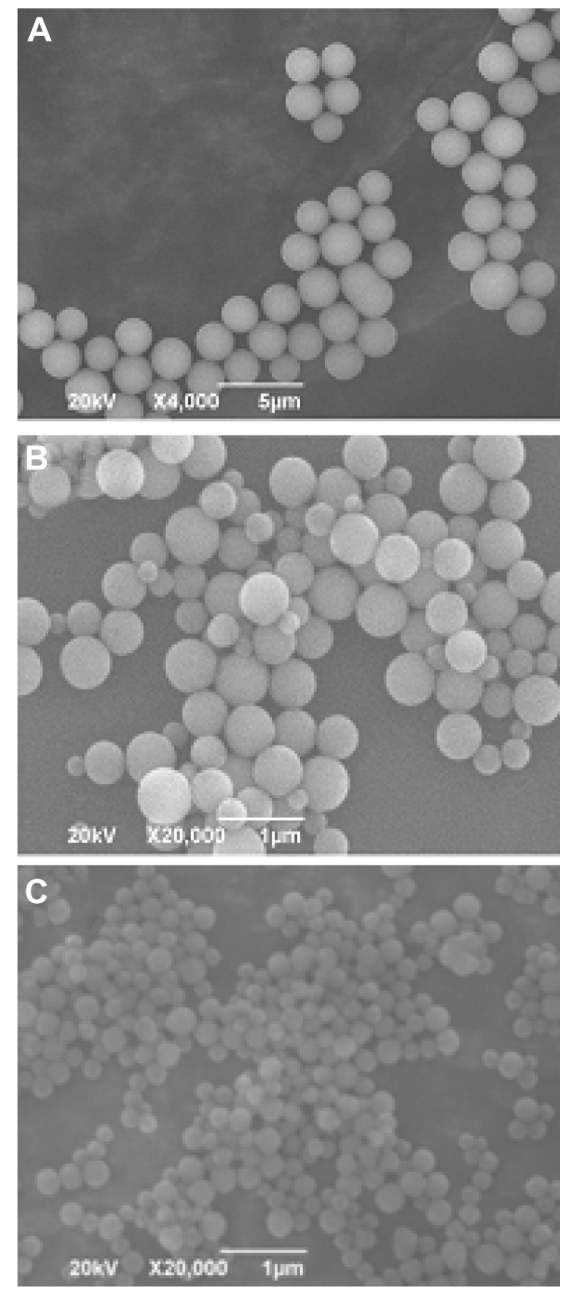

Figure 2 Scanning electron microscope images of silica nanoparticles using 3-mercaptopropyltrimethoxysilane as the sole silica source. Preparation conditions of the silica nanoparticles in (A), (B), and (C) correspond to conditions (I), (2) and (3) listed in Table I. The mean diameters of the silica nanoparticles in (A), (B), and $(\mathbf{C})$ are $2111 \mathrm{~nm}, 508 \mathrm{~nm}$ and $212 \mathrm{~nm}$, respectively.

spherical with a smooth surface and are well dispersed in water. In this synthesis process, ammonia hydrolyzes the silica precursor of MPS, and the carbon-oxygen bonds are broken to form oxygen $=$ oxygen bonds between the MPS molecules, and finally linked with each other to form a spherical grid-like structure. This structure consists of tetrahedral crystals. Each tetrahedral crystal has three silicon-oxygen residues and one mercaptopropyl group. These thiol groups are equally distributed in the inner and outer surfaces of silica NPs. This unique property will be very helpful for further modification and for doping a fluorescent dye through the chemical reaction of thiol and maleimide groups. Note that the ammonia concentration strongly affects the rate of reaction and the stability of the reaction system. It was found that a high ammonia concentration decreased the stability of the reaction systems, and led to NPs with an irregular shape (data not shown).

\section{Preparation, medication, and characterization of fluorescent NPs}

The fluorescent dye was also conjugated to the silica NPs surfaces (NPs-FITC), or directly doped into the silica NPs (NPs[FITC]) during the NPs preparation through the covalent reaction of thiol and maleimide groups. Using fluorescence microscopy, we compared the fluorescence intensities of NPs-FITC and NPs(FITC) particles in $\mathrm{ddH}_{2} \mathrm{O}$ solution at the same particle density. Although the two types of NPs exhibited well-dispersed and distinct fluorescence, the fluorescence intensity of NPs(FITC) was significantly higher than that of NPs-FITC as shown in Figure 3. This finding indicated that FITC internally doped is better than FITC surfaceconjugated, which suggested that more FITC molecules were doped into the NPs(FITC). Furthermore, FITC molecules were stably doped into the alkoxysilane backbone of the silica NPs and could not leak from the NPs(FITC) owing to the formation of MPS-FITC conjugate during preparation. The high fluorescence intensity of NPs(FITC) gives them high potential for use as a fluorescent nanoprobe in bioimaging, bioassay, and nanomedicine.

Fluorescence spectroscopy and FT-IR spectroscopy were used to determine whether folate molecules were modified to conjugate to the NPs(FITC) surfaces. The fluorescence emission spectra exhibited a fluorescence excitation peak at a wavelength of approximately $440 \mathrm{~nm}$ (Figure 4), which is the characteristic fluorescence emission peak of folate molecules. ${ }^{18,19}$ This suggests that folate may be successfully conjugated to the NPs(FITC) surfaces. To further verify this, FT-IR spectroscopy analysis was performed to confirm the presence of folate-grafted NPs(FITC). Figure 5 shows a comparison between the FT-IR absorbance spectra of NPs(FITC) and NPs(FITC)-PEG-Folate. After the NPs(FITC) were modified by PEG-Folate, two clear absorbance peaks were observed at wavenumbers of $1500 \mathrm{~cm}^{-1}$ and $1657 \mathrm{~cm}^{-1}$, which correspond to the absorbance of the benzene loop backbone
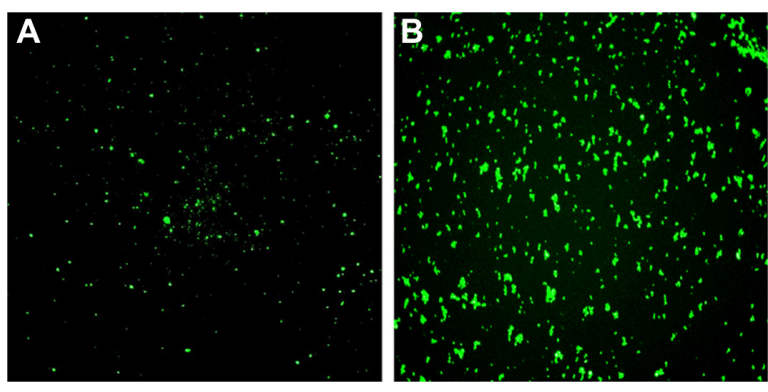

Figure 3 Fluorescence microscopy of nanoparticles-fluorescein isothiocyanate (NPs-FITC) and NPs(FITC) in solution. The sizes for the two types of particles are $212 \mathrm{~nm}$ (magnification 600X). 

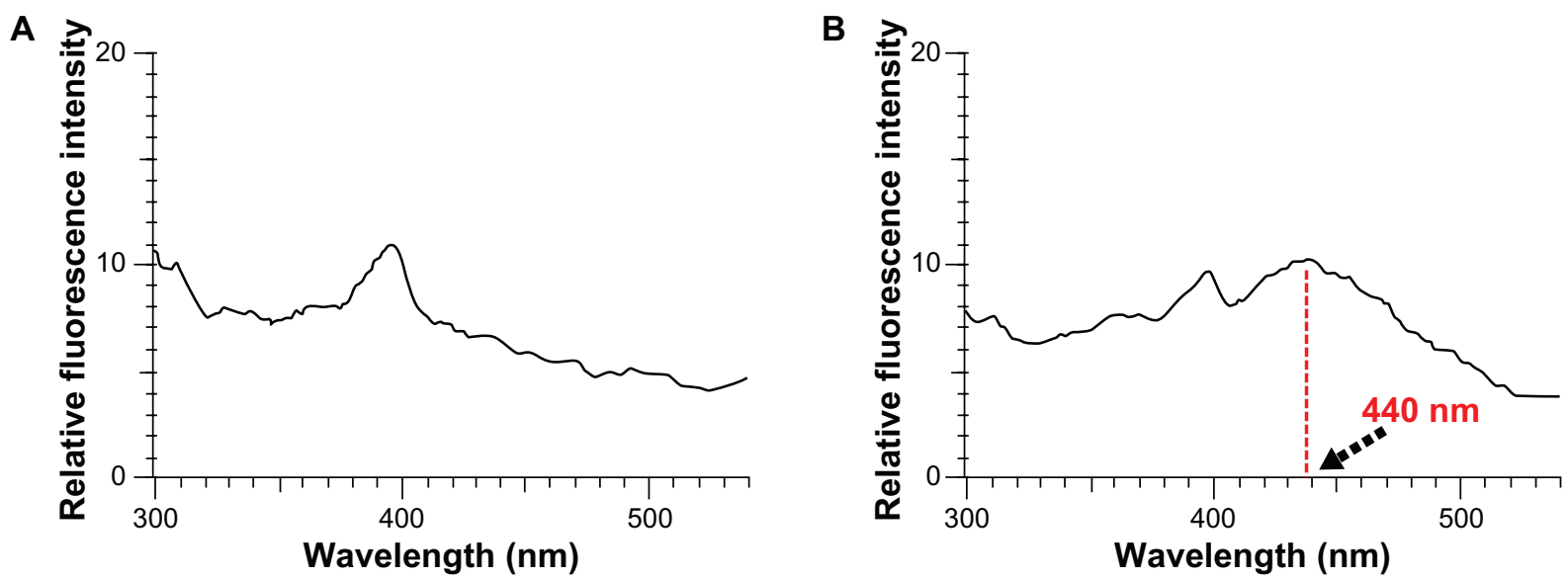

Figure 4 Fluorescence emission spectra of nanoparticles-(fluorescein isothiocyanate) (NPs[FITC]) particles (A) and NPs(FITC)-polyethylene glycol-Folate particles (B). The fluorescence excitation peak at a wavelength of approximately $440 \mathrm{~nm}$ indicates successful coupling of folate to particle surfaces.

of folate and the amide bond (-CO-NH-). The presence of the amide bond demonstrated that PEG-Folate conjugation was successful because $\mathrm{NH}_{2}$-PEG-maleimide reacted with folate to form the amide bonds. Taken together, the results of fluorescence spectroscopy and FT-IR spectroscopy confirmed the presence of PEG-Folate on the NPs(FITC) surfaces, and thus successfully engineered novel NPs(FITC)-PEGFolate particles. To investigate the zeta potential after PEGFolate modification, the zeta potentials of NPs(FITC) and NPs(FITC)-PEG-Folate were compared in $\mathrm{ddH}_{2} \mathrm{O}$ solution. It was found that the zeta potential was hardly changed after PEG-Folate coupling (Figure 6), which suggests that PEG-Folate coupling does not change the surface charge of NPs(FITC) particles.

\section{Cellular internalization of targeted and nontargeted fluorescent NPs}

To further investigate and evaluate the potential biomedical applications of NPs(FITC) and NPs(FITC)-PEG-Folate, their selective cellular uptake (or cellular internalization) in $\mathrm{KB}$ cells via an inverted fluorescence microscope and confocal microscope were compared. It was found that few

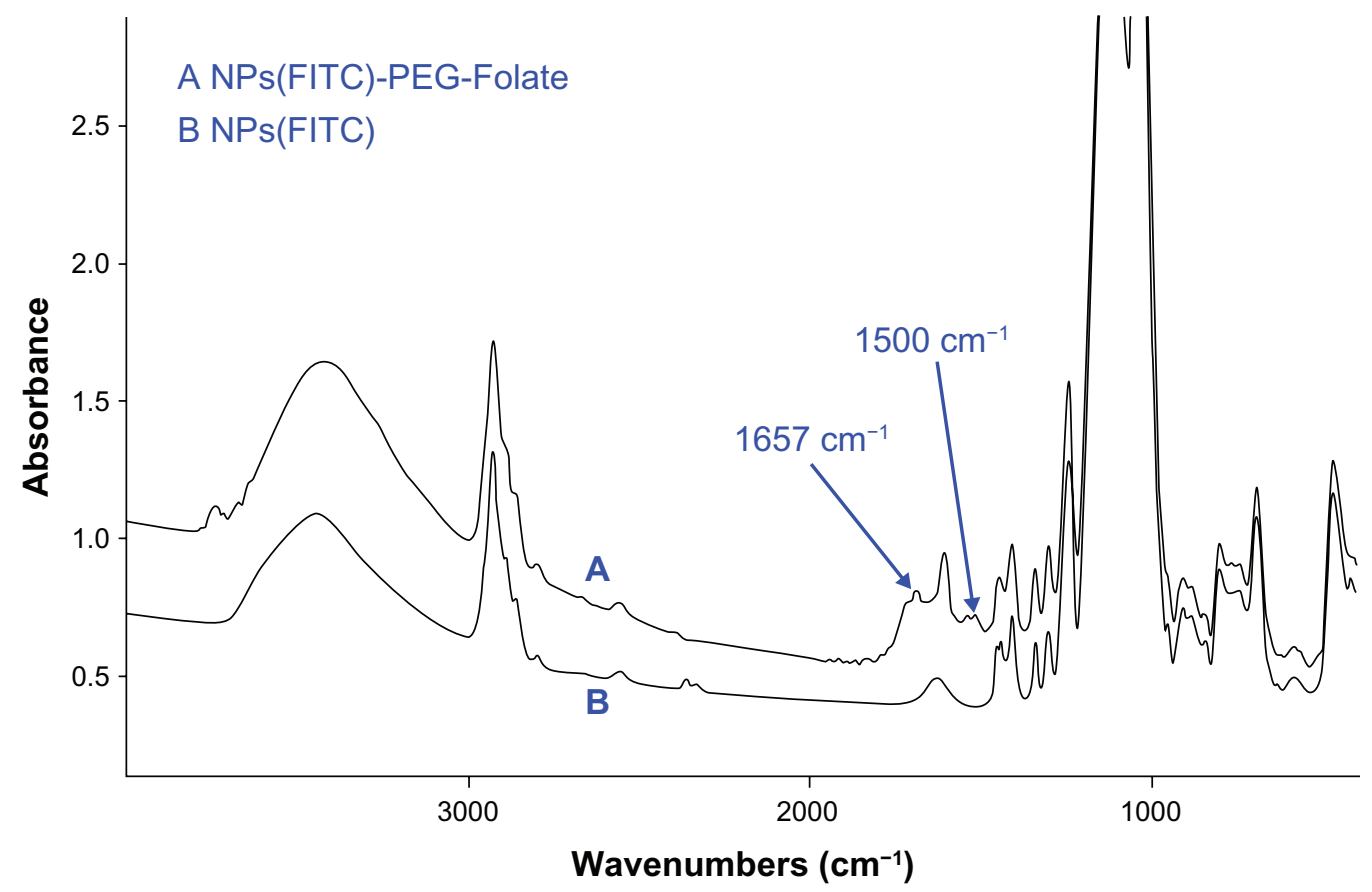

Figure 5 Fourier transform infrared spectra of nanoparticles-(fluorescein isothiocyanate)-polyethylene glycol-Folate (NPs[FITC]-PEG-Folate) particles (A) and NPs(FITC) particles (B). 

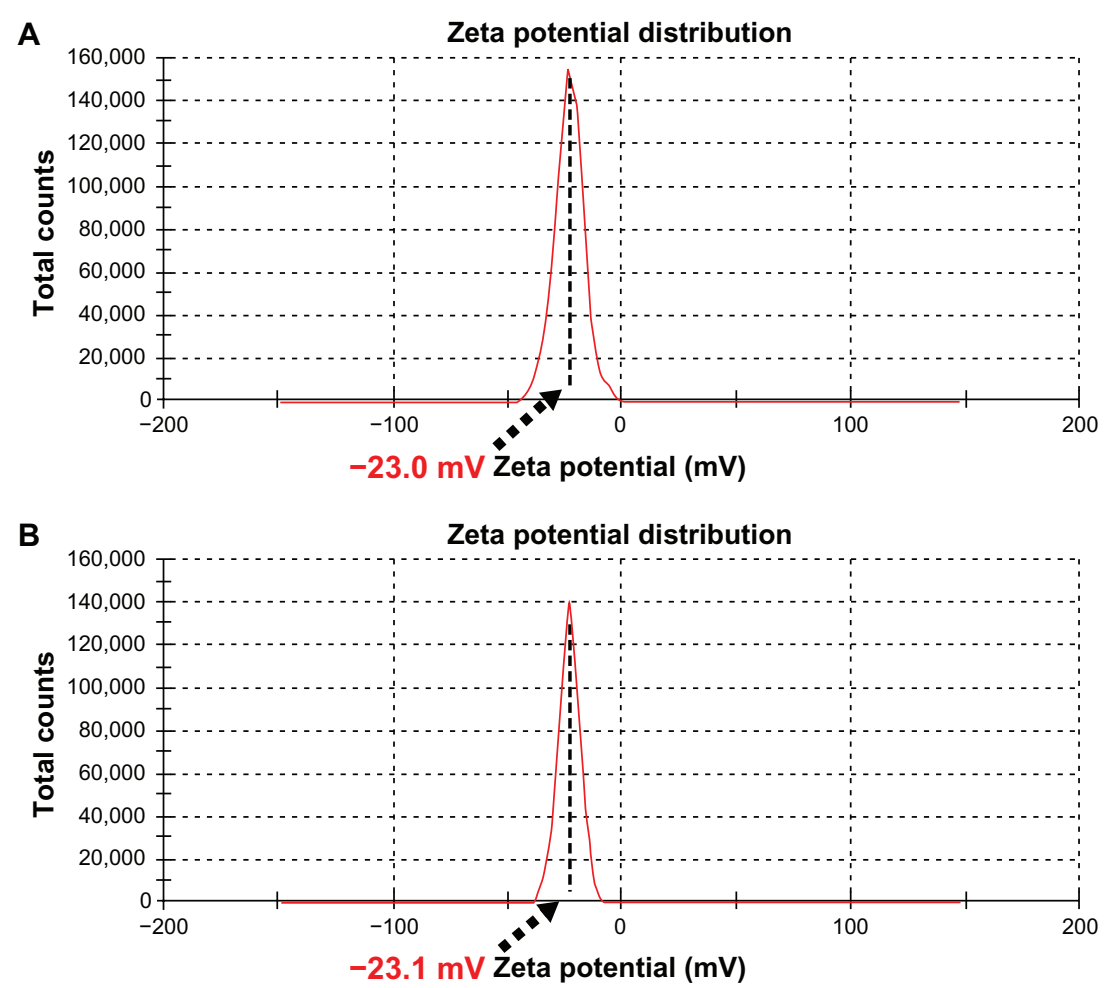

Figure 6 Zeta potential measurements. (A) The zeta potential of unmodified nanoparticles-(fluorescein isothiocyanate) (NPs[FITC]) particles was $-23.0 \mathrm{mV}$. (B) The zeta potential of NPs(FITC)-polyethylene glycol-Folate particles was $-23.1 \mathrm{mV}$.

NPs(FITC) were internalized into the KB cells because little fluorescence was observed, as shown in Figure 7A-C. In contrast, NPs(FITC)-PEG-Folate were easily taken up by $\mathrm{KB}$ cells (Figure 7D-F). Because of the overexpression of the folate receptor by KB cells, the enhanced cellular uptake of the folate-decorated NPs(FITC)-PEG-Folate may have been due to folate receptor-mediated internalization. ${ }^{20,21}$ To further determine whether these NPs were internalized into the KB cells, confocal laser scanning microscopy was used to visualize the NPs in cells. After incubating NPs(FITC) or

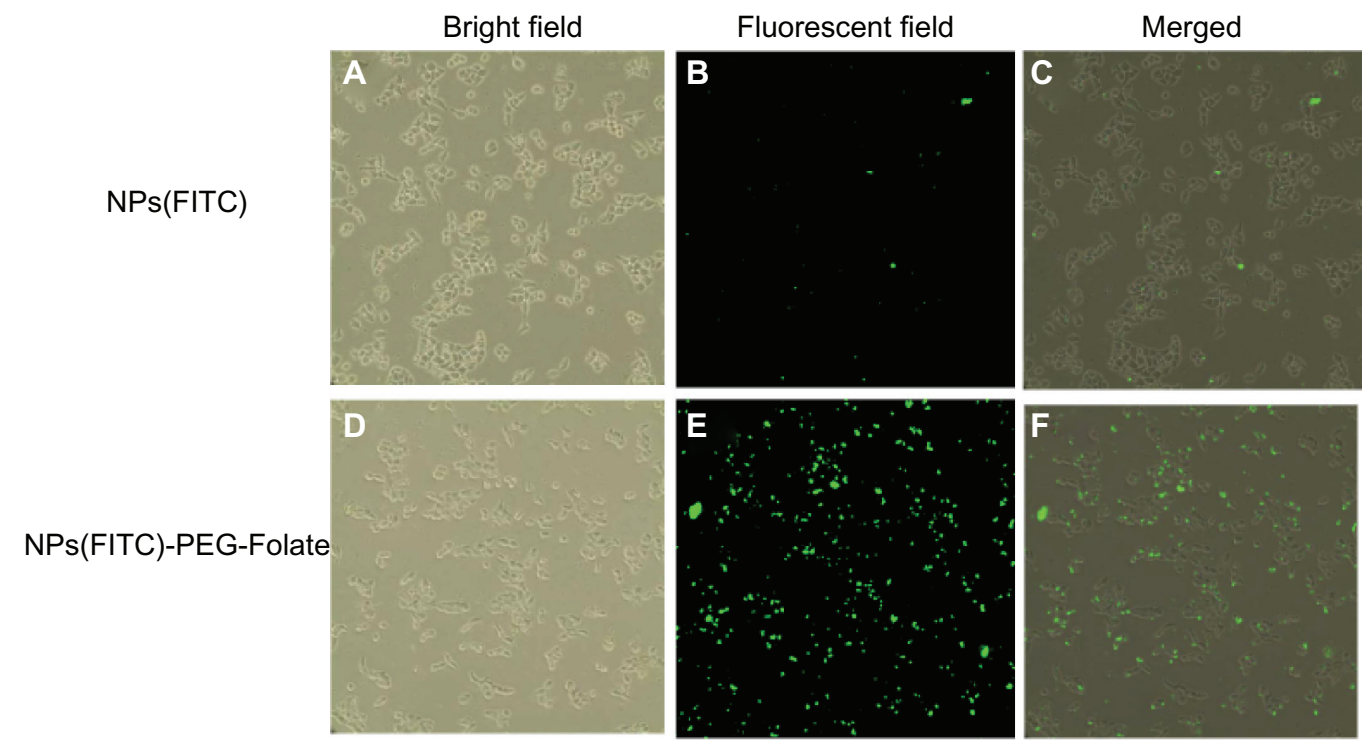

Figure 7 Evaluation of cellular uptake (internalization). Nontargeted nanoparticles-(fluorescein isothiocyanate) (NPs[FITC]) particles (upper panels) and targeted NPs(FITC)polyethylene glycol(PEG)-Folate particles (lower panels) were incubated with $\mathrm{KB}$ cells for 6 hours at $37^{\circ} \mathrm{C}$, then washed three times with phosphate buffer solution and observed under an inverted fluorescence microscope (magnification 200×). 
NPs(FITC)-PEG-Folate with $\mathrm{KB}$ cells for 6 hours at $37^{\circ} \mathrm{C}$ and removing excess NPs, it was also observed a large number of NPs(FITC)-PEG-Folate in a punctuate pattern inside the targeted cells (Figure 8B), suggesting an efficient targeting and internalization mechanism for $212 \mathrm{~nm}$ NPs(FITC)-PEGFolate to folate receptor-positive cells. In contrast, untargeted NPs(FITC) particles were only slightly internalized by KB cells after the same duration of incubation (Figure 8A). These findings were in good agreement with the observation via inverted fluorescence microscopy. It was also found that the PEG spacer has little effect on the NPs cellular uptake (data not shown). Taking these results together, the folate receptor-mediated internalization of NPs(FITC)-PEG-Folate was corroborated.

The quantitative analysis and evaluation of NPs cellular internalization were performed by flow cytometry, the results of which are shown in Figure 9. Histograms showing the cellular internalization of A549 and KB cells incubated with NPs(FITC) or NPs(FITC)-PEG-Folate for 6 hours are used to display the flow cytometry data, which showed that cellular internalization was clearly different for A549 cells (folate receptor-negative cells) and $\mathrm{KB}$ cells (folate receptor-positive cells). Particle cellular internalization was very low (less than 4\%) for both NPs(FITC) and NPs(FITC)-PEG-Folate in A549 cells. It is reasonable to assume that folate coupling to particle surfaces will not affect the cellular internalization because there are no folate receptors on A549 cells. However, for the folate receptor-positive $\mathrm{KB}$ cells, significant internalization of NPs(FITC)-PEG-Folate was observed (more than $25 \%$ ), which was more than six-fold higher than that for A549 cells. It is of interest that the cellular internalization markedly decreased in the presence of $1 \mathrm{mM}$ free folate, ie, the free folate reduced the cell uptake of NPs(FITC)-PEGFolate particles. One possible explanation for this is that free folate molecules competitively bind to the folate receptors on the membrane of $\mathrm{KB}$ cells, thus reducing the possibility of binding for NPs(FITC)-PEG-Folate and KB cells.
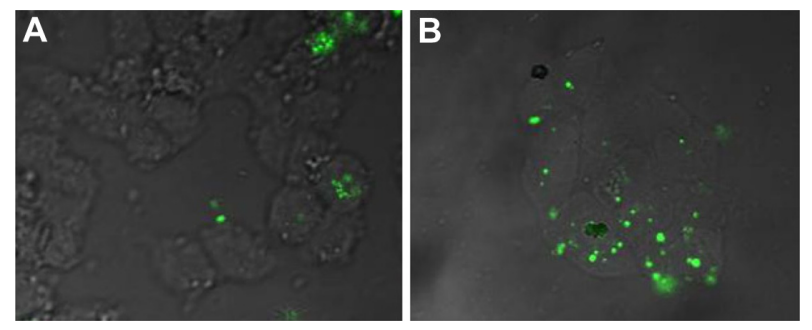

Figure 8 Confocal laser scanning microscopy images of KB cells after a 6 hour incubation period at $37^{\circ} \mathrm{C}$ with nontargeted nanoparticles-(fluorescein isothiocyanate) (NPs[FITC]) (A) and targeted NPs(FITC)-polyethylene glycol-Folate (B) (magnification 400 $\times$ ).

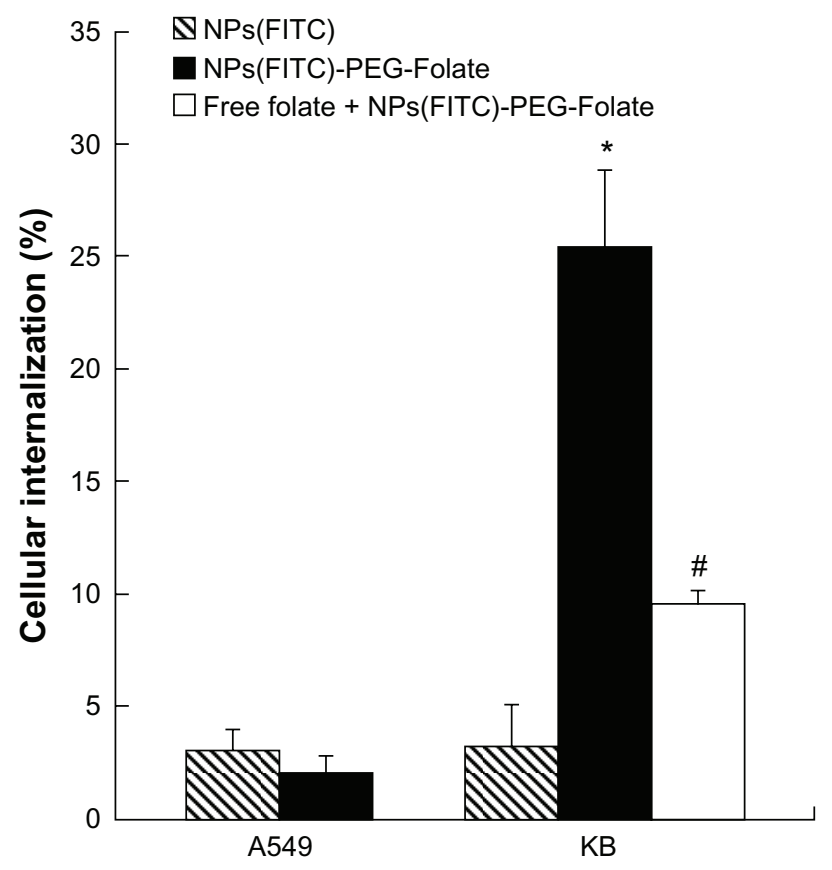

Figure 9 Cellular internalization of nanoparticles-(fluorescein isothiocyanate) (NPs[FITC]) and NPs(FITC)-polyethylene glycol(PEG)-Folate particles. All experiments were carried out with 6 hour incubation with A549 or KB cells, followed by washing three times with phosphate buffer solution and detection by flow cytometry. To obtain the rightmost bar, free folate $(\mathrm{I} \mathrm{mM})$ was preincubated with $\mathrm{KB}$ cells for I hour before the incubation of $\mathrm{NPs}(\mathrm{FITC})$-PEG-Folate.

Notes: ${ }^{P} P<0.05$ vs NP(FITC) or NPs(FITC)-PEG-Folate in A549 cells or NP(FITC) in $K B$ cells; ${ }^{\#} P<0.05$ vs NPs(FITC)-PEG-Folate in $K B$ cells.

Therefore, it is suggested that NPs(FITC)-PEG-Folate was transported into KB cells via folate receptor-mediated endocytosis. Taken together, the folate-conjugated silica particles of NPs(FITC)-PEG-Folate showed a higher ability to actively target the tumor cells with overexpressed folate receptors on cell surface in comparison with folate-free silica particles of NPs(FITC).

\section{Mechanisms of fluorescent NPs cellular internalization}

It has been reported that cells can internalize materials by several endocytotic processes such as clathrin-mediated endocytosis, caveolae-mediated endocytosis, macropinocytosis, and so forth..$^{22,23}$ To more clearly delineate the role of specific endocytotic pathways involved in the cellular internalization of NPs(FITC)-PEG-Folate, KB cells were treated with known biochemical inhibitors of energy-dependent processes, clathrin-mediated endocytosis, caveolae-mediated endocytosis, and macropinocytosis (Figure 10). To investigate the energy dependence of these processes, KB cells were preincubated in the presence of sodium azide and then treated with NPs(FITC)-PEG-Folate. Sodium azide, which blocks cellular ATP synthesis, resulted in a marked decrease in the cellular 


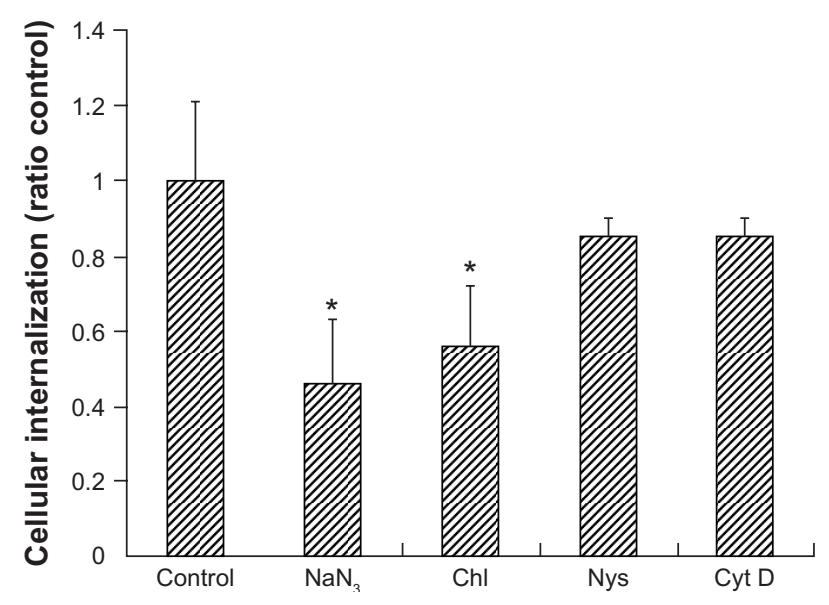

Figure 10 Investigation of the mechanisms of cellular internalization by using inhibitors of endocytosis. KB cells were incubated with the indicated inhibitors (sodium azide $\left[\mathrm{NaN}_{3}\right]$, chlorpromazine [Chl], nystatin [Nys], and cytochalasin D $[C y t D])$. The internalization ratio was normalized to that of the control (particle internalization in the absence of inhibitors).

Note: ${ }^{*} P<0.05$ vs control (no inhibitors added).

internalization (approximately 50\% decrease compared with that of nontreated cells) indicating that internalization is an energy-dependent process. Complete inhibition was not observed probably because of the presence of exogenous ATP and glucose in the serum-free media. Similarly, the internalization of NPs(FITC)-PEG-Folate was markedly decreased in the presence of $10 \mu \mathrm{g} / \mathrm{mL}$ chlorpromazine (approximately $40 \%$ decrease compared with that of nontreated cells), an inhibitor of clathrin-mediated endocytosis. However, the internalization of KB cells pretreated with nystatin (an inhibitor of caveolae-mediated endocytosis) and cytochalasin D (an inhibitor of macropinocytosis) remained approximately the same as that of the control. On the basis of the above experimental results, the authors propose that the admission of NPs(FITC)-PEG-Folate into KB cells is predominated by clathrin-mediated endocytosis and requires consumption of ATP. Note that none of the specific chemical inhibitors led to greater than $90 \%$ inhibition of internalization. This observation indicates that other pathways for internalization exist.

\section{Conclusion}

In summary, the authors have developed a strategy for the synthesis of functionalized particles of NPs(FITC)-PEG-Folate, which have two functions for targeted cancer cells, ligand biorecognition targeting (folate), and fluorescent tracking (FITC). The feasibility of delivering NPs(FITC)-PEG-Folate to cancer cells was investigated and it was demonstrated that NPs(FITC)-PEG-Folate can be internalized into folate receptorpositive KB cells with relatively high efficiency. An investigation of the internalization mechanism showed that the admission of NPs(FITC)-PEG-Folate into KB cells is predominated by clathrin-mediated endocytosis and requires the consumption of ATP. These results demonstrated that the novel particles of NPs(FITC)-PEG-Folate are promising for fluorescent imaging or targeting delivery to folate receptor-positive tumors.

\section{Acknowledgments}

The financial support provided, in whole or in part, by the National Natural Science Foundation of China (81071257), the New Century Excellent Talents Program in Chinese Universities (NCET-09-0263), the Sichuan Youth Science and Technology Foundation of China (2010JQ0004), the Fundamental Research Funds for Central Universities (23801135, 23801131), and the Visiting Scholar Foundation of Key Lab of Biorheological Science and Technology in Chongqing University, is greatly appreciated.

\section{Disclosure}

The authors report no conflicts of interest in this work.

\section{References}

1. Alivisatos P. The use of nanocrystals in biological detection. Nat Biotechnol. 2004;22(1):47-52.

2. Derfus AM, Chen AA, Min DH, Ruoslahti E, Bhatia SN. Targeted quantum dot conjugates for siRNA delivery. Bioconjug Chem. 2007;18(5): 1391-1396.

3. Nie Y, Zhang Z, Li L, Luo K, Ding H, Gu Z. Synthesis, characterization and transfection of a novel folate-targeted multipolymeric nanoparticles for gene delivery. J Mater Sci Mater Med. 2009;20(9): 1849-1857.

4. Heitsch AT, Smith DK, Patel RE, Ress D, Korgel BA. Multifunctional particles: magnetic nanocrystals and gold nanorods coated with fluorescent dye-doped silica shells. J Solid State Chem. 2008;181(7): 1590-1599.

5. Bharali DJ, Klejbor I, Stachowiak EK, et al. Organically modified silica nanoparticles: a nonviral vector for in vivo gene delivery and expression in the brain. Proc Natl Acad Sci U S A. 2005;102(321):11539-11544.

6. Choi J, Zheng Q, Katz HE, Guilarte TR. Silica-based nanoparticle uptake and cellular response by primary microglia. Environ Health Perspect. 2010;118(5):589-595.

7. Nakamura M, Ishimura K. Synthesis and characterization of organosilica nanoparticles prepared from 3-mercaptopropyltrimethoxysilane as the single silica source. J Phys Chem C. 2007;111(51):18892-18898.

8. Nakamura M, Ishimura K. One-pot synthesis and characterization of three kinds of thiol-organosilica nanoparticles. Langmuir. 2008;24(9):5099-5108.

9. Nakamura M, Shono M, Ishimura K. Synthesis, characterization, and biological applications of multifluorescent silica nanoparticles. Anal Chem. 2007;79(17):6507-6514.

10. Koch AM, Reynolds F, Kircher MF, Merkle HP, Weissleder R, Josephson L. Uptake and metabolism of a dual fluorochrome Tatnanoparticle in HeLa cells. Bioconjug Chem. 2003;14(6):1115-1121.

11. Pirollo KF, Rait A, Zhou Q, et al. Materializing the potential of small interfering RNA via a tumor-targeting nanodelivery system. Cancer Res. 2007;67(7):2938-2943.

12. Zhang Y, Guo L, Roeske RW, Antony AC, Jayaram HN. Pteroylgamma-glutamate-cysteine synthesis and its application in folate receptor-mediated cancer cell targeting using folate-tethered liposomes. Anal Biochem. 2004;332(1):168-177. 
13. Yang X, Koh CG, Liu S, et al. Transferrin receptor-targeted lipid nanoparticles for delivery of an antisense oligodeoxyribonucleotide against Bcl-2. Mol Pharm. 2009;6(1):221-230.

14. Han X, Liu J, Liu M, et al. 9-NC-loaded folate-conjugated polymer micelles as tumor targeted drug delivery system: preparation and evaluation in vitro. Int $J$ Pharm. 2009;372(1-2):125-131.

15. Lee ES, Na K, Bae YH. Polymeric micelle for tumor $\mathrm{pH}$ and folatemediated targeting. J Control Release. 2003;91(1-2):103-113.

16. Zhao H, Yung LY. Selectivity of folate conjugated polymer micelles against different tumor cells. Int J Pharm. 2008;349(1-2):256-268.

17. Hilgenbrink AR, Low PS. Folate receptor-mediated drug targeting: from therapeutics to diagnostics. J Pharm Sci. 2005;94(10):2135-2146.

18. Santra S, Kaittanis C, Grimm J, Perez JM. Drug/dye-loaded, multifunctional iron oxide nanoparticles for combined targeted cancer therapy and dual optical/magnetic resonance imaging. Small. 2009; 5(16): 1862-1868.
19. Zou T, Li SL, Cheng SX, Zhang XZ, Zhuo RX. Synthesis of poly(alpha, beta-[N-(2-hydroxyethyl)-L-aspartamide])-folate for drug delivery. J Biomater Sci Polym Ed. 2010;21(6):759-770.

20. Esmaeili F, Ghahremani MH, Ostad SN, et al. Folate-receptor-targeted delivery of docetaxel nanoparticles prepared by PLGA-PEG-folate conjugate. J Drug Target. 2008;16(5):415-423.

21. Kim SH, Jeong JH, Chun KW, Park TG. Target-specific cellular uptake of PLGA nanoparticles coated with poly(L-lysine)-poly(ethylene glycol)-folate conjugate. Langmuir. 2005;21(19):8852-8857.

22. Gratton SE, Ropp PA, Pohlhaus PD, et al. The effect of particle design on cellular internalization pathways. Proc Natl Acad Sci U SA. 2008;105(33):11613-11618.

23. Zhang X, Meng L, Wang X, Lu Q. Preparation and cellular uptake of $\mathrm{pH}-$ dependent fluorescent single-wall carbon nanotubes. Chemistry. 2010;16(2):556-561.
International Journal of Nanomedicine

\section{Publish your work in this journal}

The International Journal of Nanomedicine is an international, peerreviewed journal focusing on the application of nanotechnology in diagnostics, therapeutics, and drug delivery systems throughout the biomedical field. This journal is indexed on PubMed Central, MedLine, CAS, SciSearch ${ }^{\circledR}$, Current Contents ${ }^{\circledR} /$ Clinical Medicine,

\section{Dovepress}

Journal Citation Reports/Science Edition, EMBase, Scopus and the Elsevier Bibliographic databases. The manuscript management system is completely online and includes a very quick and fair peer-review system, which is all easy to use. Visit http://www.dovepress.com/ testimonials.php to read real quotes from published authors. 